\title{
Early prediction of long-term upper limb spasticity after stroke
}

\author{
Part of the SALGOT study \\ OPEN
}

Arve Opheim, PT, PhD

Anna Danielsson, PT,

$\mathrm{PhD}$

Margit Alt Murphy, PT, $\mathrm{PhD}$

Hanna C. Persson, PT, $\mathrm{MSc}$

Katharina Stibrant Sunnerhagen, MD, $\mathrm{PhD}$

Correspondence to Dr. Opheim: arve.opheim@neuro.gu.se

Supplemental data at Neurology.org

\section{ABSTRACT}

Objective: To identify predictors and the optimal time point for the early prediction of the presence and severity of spasticity in the upper limb 12 months poststroke.

Methods: In total, 117 patients in the Gothenburg area who had experienced a stroke for the first time and with documented arm paresis day 3 poststroke were consecutively included. Assessments were made at admission and at 3 and 10 days, 4 weeks, and 12 months poststroke. Upper limb spasticity in elbow flexion/extension and wrist flexion/extension was assessed with the modified Ashworth Scale (MAS). Any spasticity was regarded as MAS $\geq 1$, and severe spasticity was regarded as MAS $\geq 2$ in any of the muscles. Sensorimotor function, sensation, pain, and joint range of motion in the upper limb were assessed with the Fugl-Meyer assessment scale, and, together with demographic and diagnostic information, were included in both univariate and multivariate logistic regression analysis models. Seventy-six patients were included in the logistic regression analysis.

Results: Sensorimotor function was the most important predictor both for any and severe spasticity 12 months poststroke. In addition, spasticity 4 weeks poststroke was a significant predictor for severe spasticity. The best prediction model for any spasticity was observed 10 days poststroke (85\% sensitivity, 90\% specificity). The best prediction model for severe spasticity was observed 4 weeks poststroke (91\% sensitivity, 92\% specificity).

Conclusions: Reduced sensorimotor function was the most important predictor both for any and severe spasticity, and spasticity could be predicted with high sensitivity and specificity 10 days poststroke. Neurology ${ }^{\circledR}$ 2015;85:873-880

\section{GLOSSARY}

ADL = activities of daily living; ARAT = Action Research Arm Test $\mathbf{C l}=$ confidence interval; FMA-UE = Fugl-Meyer Assessment Upper Extremity Scale; MAS = modified Ashworth Scale; NIHSS = NIH Stroke Scale; NLR = negative likelihood ratio; PLR = positive likelihood ratio; ROM = range of motion; SALGOT = Stroke Arm Longitudinal Study at the University of Gothenburg.

Upper limb spasticity has been found to be associated with reduced arm function and low levels of independence, and with a 4-fold increase in direct care costs during the first year poststroke. ${ }^{1-5}$ The prevalence of upper limb spasticity in all patients 12 months poststroke varies from $17 \%$ to $38 \%{ }^{6-10}$ and was found to be $46 \%$ in patients with initial impaired arm function. ${ }^{5}$ It has been found that $4 \%-$ $13 \%$ of patients need treatment for spasticity 6-12 months poststroke. ${ }^{6,9}$ Previous studies during the first 10 days poststroke have identified several predictors for spasticity 3-12 months poststroke, e.g., reduced sensorimotor function and activities of daily living (ADL), muscle weakness, left-sided paresis, and smoking. ${ }^{8,911,12}$ These studies were relatively small $(\mathrm{n}=47),{ }^{9}$ assessed patients several days after stroke onset, ${ }^{11}$ or assessed spasticity in both upper and lower limbs. Whether early assessments of upper limb function and impairments can predict the occurrence and degree of upper limb spasticity 12 months poststroke with good accuracy is uncertain. The optimal time for early prediction of upper limb spasticity 12 months poststroke is also unknown. ${ }^{12}$ This information is of clinical relevance, as patients with an increased risk of developing spasticity-related impairments, complications, and

\footnotetext{
From the Institute of Neuroscience and Physiology (A.O., A.D., M.A.M., H.C.P., K.S.S.), Rehabilitation Medicine, Sahlgrenska Academy, University of Gothenburg, Sweden; and Sunnaas Rehabilitation Hospital (A.O.), Nesoddtangen, Norway.

Go to Neurology.org for full disclosures. Funding information and disclosures deemed relevant by the authors, if any, are provided at the end of the article. The Article Processing Charge was paid by the authors.

This is an open access article distributed under the terms of the Creative Commons Attribution License 4.0 (CC BY), which permits unrestricted use, distribution, and reproduction in any medium, provided the original work is properly cited.
} 
increased disability may be identified. ${ }^{1,13}$ The study aims were to identify predictor variables and the optimal time for early prediction of any spasticity and severe spasticity in the upper limb 1 year poststroke.

METHODS All patients with first-ever stroke in an 18-month period in 2009-2010 who were admitted to the largest of 3 acute stroke units at the Sahlgrenska University Hospital, Gothenburg, Sweden, within 3 days after stroke onset were eligible for consecutive screening for inclusion in the present study, which was a part of the Stroke Arm Longitudinal Study at the University of Gothenburg (SALGOT). In the SALGOT study, the recovery of upper extremity function was investigated in a nonselected sample during the first year poststroke. ${ }^{14}$ All included patients had ischemic or hemorrhagic stroke ${ }^{15}$ for the first time, were over 18 years old, and had impaired upper extremity function, which was assessed at day 3 with the Action Research Arm Test (ARAT) $(0-57)^{16}$ and defined as $<57$ points. The study sample size estimation $(\mathrm{n}=88)$ for SALGOT was to determine a medium change of 6 points (10\%) on ARAT, with a power of 0.8 and a significance level of 0.05 . With an expected dropout rate of $30 \%$, the aim was to include 120 patients. ${ }^{14}$

Standard protocol approvals, registrations, and patient consents. Study approval was provided by the Regional Ethics Committee of the Western region of Sweden (Registration number 225/08), and written informed consent was obtained. The study is registered at www.clinicaltrials.gov (NCT 01115348).

Assessment procedure. In SALGOT, the patients were assessed 9 times during the first year: at admission; at 3 and 10 days; at 3, 4, and 6 weeks; and at 3, 6 and 12 months poststroke. In the current study, data from admission, 3 and 10 days, 4 weeks, and 12 months were used. Predominantly, the assessments were carried out by 3 physiotherapists and were performed according to a standardized protocol. ${ }^{14} \mathrm{~A}$ majority of the assessments were performed at the university hospital. If traveling was not possible for the patient, the assessments were conducted in the patient's home, nursing home, or rehabilitation unit.

Variables. Predictor variables collected at admission (day 0). Clinical characteristics and assessments routinely registered at admission during the acute stage of stroke were selected as potential predictor variables (age, sex, ischemic or hemorrhagic stroke, side of stroke, and smoking in the last 3 months). Stroke localization was classified using the Oxfordshire Classification ${ }^{17}$ and ischemic stroke was classified after cause of lesion using the Trial of Org 10172 in Acute Stroke Treatment criteria. ${ }^{18}$ The initial severity of stroke and arm paresis was assessed with the 0 - to 42point ordinal NIH Stroke Scale (NIHSS) and the NIHSS arm subscale (0-4), respectively. ${ }^{19}$ NIHSS arm was treated as a categorical variable with 0 as the reference category.

Predictor variables collected 3 and 10 days and 4 weeks poststroke. Common clinical assessment scales of sensorimotor impairments assessed at 3 and 10 days and 4 weeks poststroke were selected as potential predictors. Sensorimotor function in the upper limb was assessed with the motor function part (sections A-D) of the Fugl-Meyer Assessment Upper Extremity Scale (FMA-UE). ${ }^{20}$ The FMA-UE (sections A-D) includes 33 active motor function tests, where a higher score indicates a better performance (0-66). The nonmotor domains of the same scale (sections $\mathrm{H}-\mathrm{J}$ ) were used to assess sensation (0-12), joint pain (0$24)$, and range of motion (ROM) during passive joint motions
(0-24); lower scores indicate reduced sensation, more pain, and reduced ROM, respectively. Spasticity in elbow flexors, elbow extensors, wrist flexors, and wrist extensors were assessed with the 6-level modified Ashworth Scale (MAS). ${ }^{21}$ The original MAS categories were reordered into integers between 0 and 5, to incorporate the score $1+$. MAS were dichotomized, and spasticity was considered to be present if the MAS score was $\geq 1$ in any of these muscle groups.

Dependent variables. At 12 months poststroke, spasticity was reassessed in a similar way as previously. Any spasticity was considered if the MAS score was $\geq 1$, and severe spasticity was considered if the MAS score was $\geq 2$ in any of the muscle groups. $^{22}$

Statistical methods. Continuous and normally distributed variables are presented with means and SDs. Ordinal and nonnormally distributed variables are presented with medians and 1st and 3rd quartile (Q1-Q3). Univariate logistic regression analyses were used to assess the relationship between the potential predictor variables and the outcome variable. The predictor variables were tested for correlations, and when 2 variables had a high correlation $(r>0.8)$, one was omitted before multivariate logistic regression analysis. The multivariate logistic regression analyses were used to predict the presence of (A) any spasticity and (B) severe spasticity at 12 months poststroke. In both (A) and (B), 4 models (A1-A4 and B1-B4) were found and compared (figure 1). The criteria for including a potential predictor variable in the multivariate logistic regression analyses were as follows: (1) significant predictor identified in previous studies, with the condition that if these variables were not found predictive in models $\mathrm{A} / \mathrm{B} 1$, they were not included in models $\mathrm{A} /$ B2-4, and (2) univariate logistic regression $p$ value $<0.20$. Age and sex were included in all models. The variables in the multivariate logistic regression analysis are shown in figure 1 .

In the multivariate logistic regression analysis, the enter method was used stepwise and nonsignificant variables were removed manually one at the time, to ensure that only significant variables $(p<0.05)$ were included in the final model. To control for possible nonlinearity between summed ordinal predictors and the dependent variable, a multivariate model with the squared predictor variables (FMA-UE ${ }^{2}$ and Sensation ${ }^{2}$ ) was tested. Nonsignificant associations were interpreted as nonlinearity not shown. ${ }^{23}$ The results are presented with unstandardized coefficients, $p$ values, and odds ratios with $95 \%$ confidence intervals (95\% CIs). ${ }^{24}$ The sensitivity (\%), specificity (\%), positive likelihood ratio (PLR), negative likelihood ratio (NLR), and the corresponding $95 \% \mathrm{CI}$ for each of the models was calculated using MedCalc for Windows, version 12.7.7.0 (MedCalc Software, Ostend, Belgium). All other statistical calculations were performed using IBM SPSS statistics 21.0 (IBM, Armonk, NY). The Strengthening the Reporting of Observational Studies in Epidemiology (STROBE) guidelines were followed..$^{25,26}$

RESULTS Participants. In total, 763 patients were admitted to the stroke unit during the inclusion period, 117 of whom were included in the study and assessed at day 3 (SD 1). There were no significant differences between the participating and nonparticipating patients in terms of sex or NIHSS score. The nonparticipating patients were significantly older (76.0 [SD 13.1] vs 69.2 [SD 13.2] years, $p<0.001)$, and fewer of them had hemorrhagic infarction ( $6 \%$ vs $\left.16 \%\left[\chi^{2}=4.92, p=0.027\right]\right)$. 
Figure 1 The multivariate logistic regression analyses models

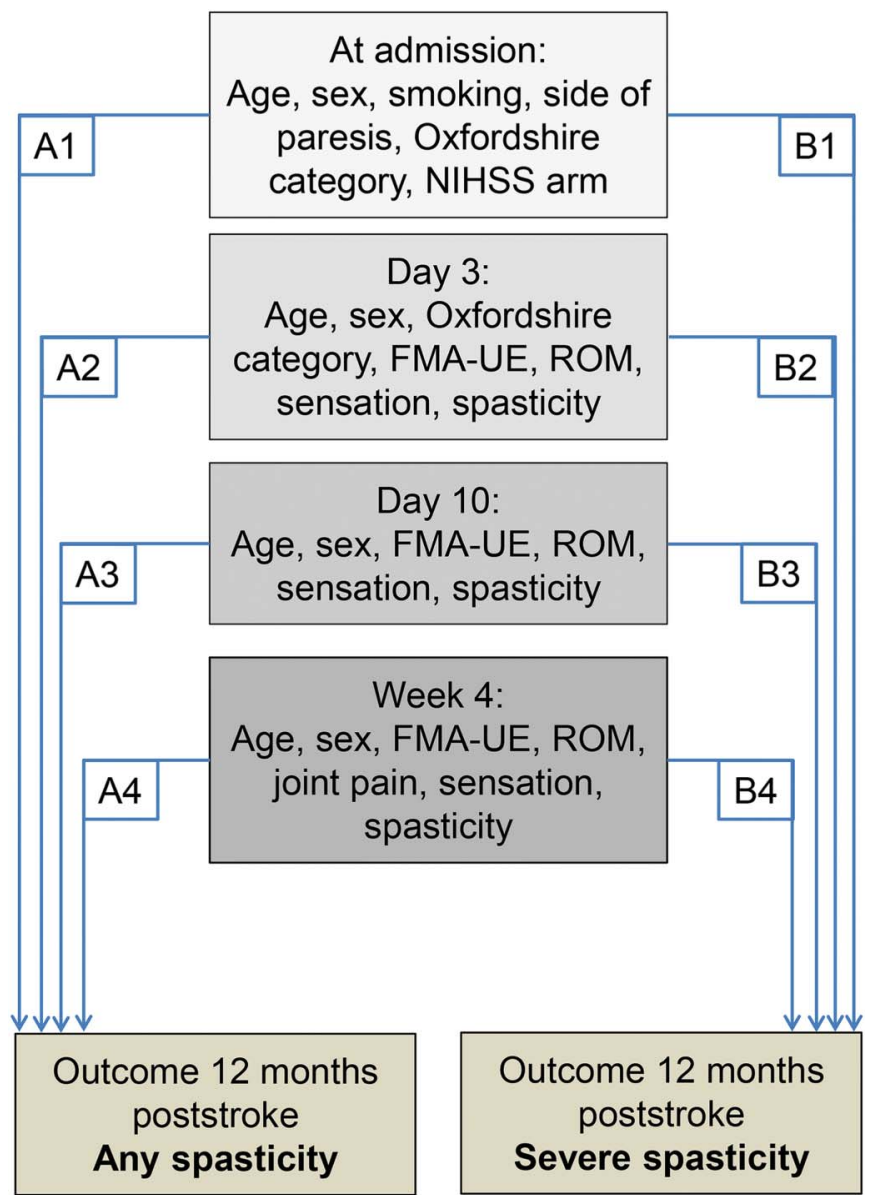

At day 3, Oxfordshire category and spasticity was included only in model B2. FMA-UE = Fugl-Meyer Assessment Upper Extremity scale; NIHSS = NIH Stroke Scale; ROM = range of motion.

In total, 76 patients were assessed 12 months poststroke (35\% dropout) and included in the logistic regression analysis. The 2 main causes for dropout were death $(\mathrm{n}=14)$ and study withdrawal $(\mathrm{n}=7)$. The clinical characteristics of the 76 patients included in the logistic regression analysis are shown in table 1.

At 3 and 10 days and 4 weeks, 24\%, 43\%, and 46\% of the 76 patients were assessed with any spasticity, respectively. At 12 months, $46 \%$ and $29 \%$ were assessed as having any spasticity and severe spasticity, respectively.

Prediction of any spasticity 12 months poststroke. The univariate logistic regression analysis for all potential predictor variables for any spasticity and severe spasticity is presented in tables e- 1 and e- 2 on the $\mathrm{Neu}$ rology ${ }^{\circledR}$ Web site at Neurology.org. The multivariate logistic regression analysis results are presented in table 2. In models $\mathrm{A} 2$ and $\mathrm{A} 3$, the FMA-UE was a significant predictor. In model A4, age at stroke onset was a significant predictor in addition to FMA-UE, and higher FMA-UE scores and higher age were associated with reduced probability for spasticity. The sensitivity, specificity, PLR, and NLR of model
A3 indicated that this model had the highest predictive value (table 3 ). The fit of prediction model A3 was assessed in a scatterplot of the predicted probabilities in relation to FMA-UE at day 10 (figure 2). The squared FMA-UE variable (FMA-UE ${ }^{2}$ ) was not significant in models A2-A4, and nonlinearity could not be shown.

Prediction of severe spasticity 12 months poststroke. The results from multivariate logistic regression models B1-B4 are shown in table 2. Sensorimotor function (FMA-UE) was a significant predictor in all models, except B1. Lower FMA-UE scores (models B2-B4) were associated with an increased predicted probability for severe spasticity. In model B2 reduced sensation and in model B 4 spasticity at 4 weeks poststroke were additional significant predictors. The sensitivity, specificity, PLR, and NLR of model B4 indicated that this model had the highest predictive value (table 3 ). The squared variables (FMA-UE ${ }^{2}$ and Sensation ${ }^{2}$ ) were not significant predictors in any of the models B2-B4, thus nonlinearity could not be shown.

DISCUSSION The present study demonstrated that any spasticity was best predicted with variables collected at day 10 poststroke and severe spasticity was best predicted with variables collected 4 weeks poststroke. Lower sensorimotor function score, as identified with the FMA-UE, consistently and significantly predicted both any spasticity and severe spasticity at 12 months poststroke. Age at stroke onset was a significant predictor 4 weeks poststroke, with higher age associated with reduced probability for spasticity. The presence of upper limb spasticity 4 weeks poststroke was a significant predictor for severe spasticity.

These findings support previous studies reporting paresis and reduced ADL function to be significant predictors for spasticity poststroke. ${ }^{8,11,12}$ However, none of the previous studies used the FMA-UE to assess sensorimotor function, and thus, a direct comparison cannot be made with those studies. Additionally, none of the former studies compared different prediction models for spasticity at 12 months poststroke based on the assessments at different time points relatively early after stroke onset, as was performed in the present study. Predicting upper limb spasticity with a relatively high accuracy based on early assessments may have high clinical relevance, as patients with an increased risk of developing spasticity-related impairments may be identified early and monitored more closely in order to implement appropriate interventions.

In the present study, patients with higher age were predicted to have reduced probability for spasticity. This finding may be in accordance with a previous 
Table 1 Demographic and clinical data of the patients included in the logistic regression analysis $(n=76)$

\begin{tabular}{ll} 
Characteristics & Values \\
M/F, \% & $60 / 40$ \\
Age, y, mean (SD) & $67.2(12.0)$ \\
Ischemic/hemorrhagic stroke, \% & $82 / 18$ \\
Paretic arm, R/L, \% & $43 / 57$ \\
\hline Side of lesion, R/L/bilateral/cerebellum, \% & $54 / 41 / 4 / 1$ \\
\hline Stroke localization, Oxfordshire classification, TAC/LAC/PAC/POC, \% & $12 / 29 / 51 / 8$ \\
\hline Ischemic stroke subtypes, TOAST (n = 62), LAA/CE/SAO/OC/UND, \% & $16 / 31 / 29 / 13 / 11$ \\
\hline NIHSS, arm, median (Q1-Q3) & $2(1-4)$ \\
\hline NIHSS, total, median (Q1-Q3) & $7(3-12)$ \\
\hline Smoking, no/yes, \% & \\
Days at stroke unit, mean (SD) & $77 / 23$ \\
Admitted to inpatient rehabilitation, $\mathbf{n}$ & $12.4(7.3)$ \\
\hline Botulinum toxin treatment for spasticity, $\mathbf{n}$ & 51
\end{tabular}

Abbreviations: $\mathrm{CE}=$ cardioembolism; $\mathrm{LAA}=$ large artery atherosclerosis; $\mathrm{LAC}=$ lacunar anterior circulation; $\mathrm{OC}=$ other determined cause; $\mathrm{PAC}=$ partial anterior circulation; $\mathrm{POC}=$ posterior circulation; $\mathrm{Q} 1-\mathrm{Q} 3=1 \mathrm{st}$ and $3 r d$ quartile; $\mathrm{SAO}=$ small vessel occlusion; TAC $=$ total anterior circulation; TOAST $=$ Trial of Org 10172 in Acute Stroke Treatment; $\mathrm{UND}=$ undetermined cause

${ }^{\text {a At admission. }}$ study $^{22}$ where more severe spasticity was found in younger patients 3 months poststroke, but not 18 months poststroke. Muscle force generation from tendon reflexes has been found to be slower and weaker with increasing age $\mathrm{e}^{27}$ and if this also is the case for tonic reflexes associated with spasticity, spastic responses may be weaker in older patients.

In the present study, there was no association between the side of weakness and spasticity 1 year after stroke, as described previously. ${ }^{12}$ There may be several reasons for this discrepancy; for instance, there were methodologic differences between the studies, as the Tone Assessment Scale was used to assess spasticity, and spasticity and weakness were assessed in both arms and legs ${ }^{12}$ as opposed to only upper limb spasticity and sensorimotor function in the present study. An association between smoking and severe spasticity has been found previously, ${ }^{12}$ which could not be confirmed here. The authors discussed that their model, which included smoking, had an event per variable of 7 , compared to the recommended minimum of $10 .{ }^{12,28}$

Model B1 showed that patients with lacunar stroke had a lower OR for severe spasticity than those with other stroke locations. Therefore, the Oxford

Table 2 Multivariate regression analysis for the prediction of any spasticity and severe spasticity at 12 months poststroke

\begin{tabular}{|c|c|c|c|c|c|c|}
\hline & Predictors & $\begin{array}{l}\text { Unstandardized } \\
\text { coefficient }\end{array}$ & p Value & OR & 95\% Cl lower & $95 \% \mathrm{Cl}$ upper \\
\hline \multicolumn{7}{|c|}{ Any spasticity } \\
\hline \multirow[t]{5}{*}{ Model A1 } & NIHSS arm, score 0 (ref) & & 0.001 & & & \\
\hline & NIHSS arm, score 1 & -0.134 & 0.888 & 0.88 & 0.14 & 5.58 \\
\hline & NIHSS arm, score 2 & 0.742 & 0.494 & 2.10 & 0.25 & 17.59 \\
\hline & NIHSS arm, score 3 & 1.030 & 0.325 & 2.80 & 0.36 & 21.73 \\
\hline & NIHSS arm, score 4 & 2.911 & 0.003 & 18.38 & 2.75 & 122.94 \\
\hline Model A2 & FMA-UE day 3 & -0.087 & $<0.001$ & 0.92 & 0.89 & 0.95 \\
\hline Model A3 & FMA-UE day 10 & -0.083 & $<0.001$ & 0.92 & 0.89 & 0.95 \\
\hline \multirow[t]{2}{*}{ Model A4 } & FMA-UE week 4 & -0.092 & $<0.001$ & 0.91 & 0.88 & 0.95 \\
\hline & Age & -0.061 & 0.044 & 0.94 & 0.89 & 0.99 \\
\hline \multicolumn{7}{|c|}{ Severe spasticity } \\
\hline \multirow[t]{4}{*}{ Model B1 } & Oxford category, TAC (ref) & & 0.027 & & & \\
\hline & Oxford category, LAC & -3.00 & 0.003 & 0.05 & 0.007 & 0.37 \\
\hline & Oxford category, PAC & -1.39 & 0.077 & 0.25 & 0.05 & 1.16 \\
\hline & Oxford category, POC & -2.30 & 0.077 & 0.10 & 0.008 & 1.29 \\
\hline \multirow[t]{2}{*}{ Model B2 } & FMA-UE day 3 & -0.128 & 0.005 & 0.88 & 0.81 & 0.96 \\
\hline & Sensation day 3 & -0.164 & 0.045 & 0.85 & 0.72 & 0.99 \\
\hline Model B3 & FMA-UE day 10 & -0.164 & 0.024 & 0.85 & 0.74 & 0.98 \\
\hline \multirow[t]{2}{*}{ Model B4 } & FMA-UE week 4 & -0.111 & 0.001 & 0.90 & 0.84 & 0.96 \\
\hline & Spasticity week 4 & 3.42 & 0.009 & 30.62 & 2.34 & 401.5 \\
\hline
\end{tabular}

Abbreviations: $\mathrm{Cl}=$ confidence interval; FMA-UE = Fugl-Meyer Assessment Upper Extremity scale; LAC = lacunar anterior circulation; $\mathrm{NIHSS}=\mathrm{NIH}$ Stroke Scale; OR = odds ratio; Oxford = Oxfordshire Stroke Classification; PAC = partial anterior circulation; POC = posterior circulation; ref = reference category; TAC $=$ total anterior circulation.

Spasticity coded as $0=$ no spasticity; $1=$ spasticity present. 


\begin{tabular}{|c|c|c|c|c|}
\hline \multirow[t]{3}{*}{ Table 3} & \multicolumn{4}{|c|}{ Predictive properties of the different models to predict any spasticity and severe spasticity 12 months poststroke $(n=76)$} \\
\hline & \multicolumn{4}{|c|}{ Any spasticity 12 mo poststroke $(95 \% \mathrm{Cl})$} \\
\hline & Model A1 admission & Model A2 day 3 & Model A3 day 10 & Model A4 week 4 \\
\hline Sensitivity, \% & $60.0(42.1-76.1)$ & $85.7(69.7-95.1)$ & $84.4(67.2-94.7)$ & $74.3(56.7-87.5)$ \\
\hline Specificity, \% & $90.2(76.9-97.3)$ & $85.4(70.8-94.4)$ & $90.6(75.0-97.9)$ & $90.0(76.3-97.2)$ \\
\hline PLR & $6.15(2.33-16.22)$ & $5.86(2.76-12.42)$ & $9.00(3.03-26.70)$ & $7.43(2.87-19.21)$ \\
\hline \multirow[t]{3}{*}{ NLR } & $0.44(0.29-0.67)$ & $0.17(0.07-0.38)$ & $0.17(0.08-0.39)$ & $0.29(0.16-0.51)$ \\
\hline & \multicolumn{4}{|c|}{ Severe spasticity 12 months poststroke $(95 \% \mathrm{Cl})$} \\
\hline & Model B1 admission & Model B2 day 3 & Model B3 day 10 & Model B4 week 4 \\
\hline Sensitivity, \% & $27.3(10.7-50.2)$ & $90.5(69.6-97.9)$ & $94.7(73.9-99.1)$ & $90.9(70.8-98.6)$ \\
\hline Specificity, \% & $94.4(84.6-98.8)$ & $92.5(81.8-97.9)$ & $86.7(73.2-94.9)$ & $92.4(81.8-97.9)$ \\
\hline PLR & $4.91(1.35-17.91)$ & $11.99(4.62-31.07)$ & $7.11(3.35-15.08)$ & $12.05(4.65-31.19)$ \\
\hline NLR & $0.77(0.59-1.00)$ & $0.10(0.03-0.39)$ & $0.06(0.01-0.41)$ & $0.10(0.03-0.37)$ \\
\hline
\end{tabular}

Abbreviations: $\mathrm{Cl}=$ confidence interval; NLR = negative likelihood ratio; PLR = positive likelihood ratio.

categories were included in model B2 to check for stroke location as a possible predictive factor. No association between stroke location and spasticity was observed in model B2.

Presence of upper limb spasticity in the assessments during the first month was not a significant predictor for any spasticity at 12 months poststroke. Spasticity at 4 weeks poststroke was a significant predictor only for severe spasticity at 12 months. There

Figure 2 Predicted probabilities for spasticity 12 months poststroke and Fugl-
Meyer Assessment Upper Extremity scale at day 10

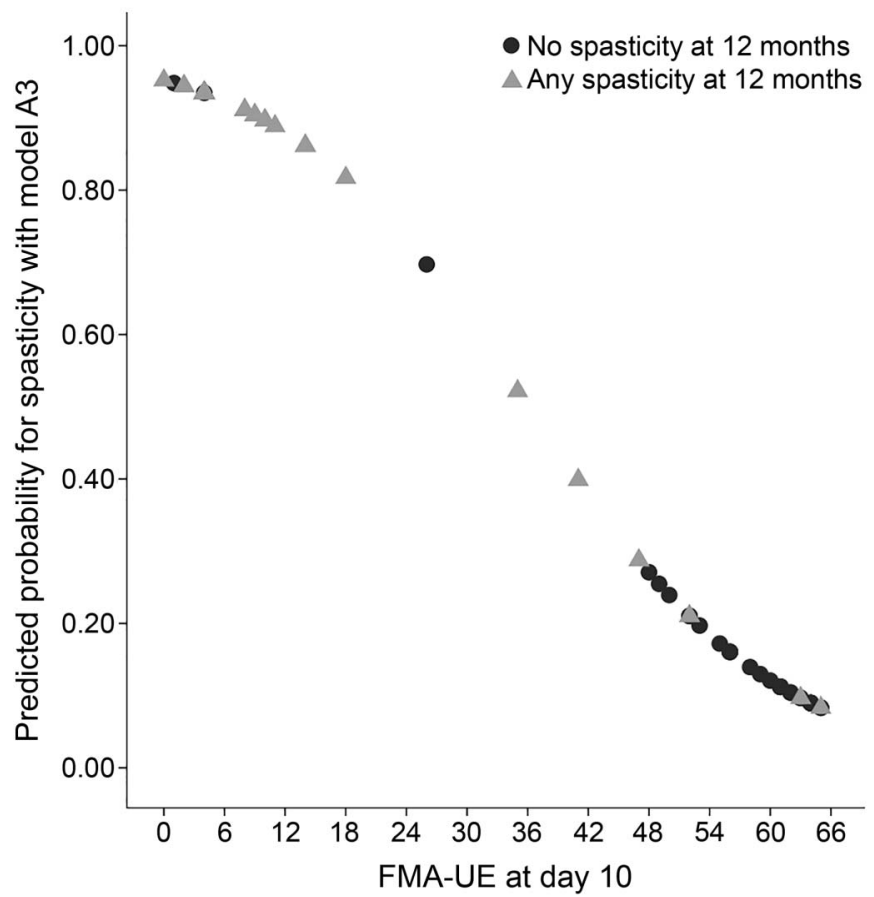

The figure shows the predicted probabilities for any spasticity at 12 months poststroke as found in model A3 (10 days poststroke), in relation to sensorimotor function at day 10, assessed with the Fugl-Meyer Assessment Upper Extremity scale (FMA-UE). may be both neurologic and muscular causes for this observation, as the tonic stretch reflexes may increase during the first 3 months, and intrinsic muscle changes may occur later. ${ }^{29}$ Therefore, spasticity may be an unstable impairment during the first months, before a more stable and manifest impairment is observed. A recent study based on the same study population supported this finding, ${ }^{5}$ as the authors found that patients changed both from no spasticity to any spasticity and vice versa during the first months poststroke.

Figure 2 shows the predicted probabilities in model $\mathrm{A} 3$ and the sensorimotor function at day 10 , and indicates a fairly good fit of the model. A perfect agreement would have resulted in a straight, negative line, with FMA-UE $=0$ equivalent to the highest probability (1.00), and FMA-UE $=66$ equivalent to the lowest probability. Those scoring $>40$ points on the FMAUE at day 10 had less than $20 \%$ probability for spasticity and those scoring $<15$ points had more than $80 \%$ probability for spasticity 12 months poststroke. There were relatively few patients scoring in the middle range (20-40 points) on the FMA-UE; therefore, the predictions of spasticity may be more uncertain in this range. The fit of model A3 was also confirmed by the relatively high sensitivity and specificity.

The clinical implications of the current study are mainly within 2 areas. First, the assessment of motor function at an early stage, either with the NIHSS at admission or with the FMA-UE, may give a good indication of the probability of a patient developing spasticity 12 months poststroke. At 3 days poststroke, the sensitivity and specificity of the prediction models were $85 \%$ and increased further at day 10 . From a clinical perspective, the assessment of sensorimotor function and the early identification of patients at risk of developing spasticity and in particular severe 
spasticity may be important. Spasticity has been found to be associated with pain, reduced range of motion, and reduced motor function, which can have a negative impact on the functional ability of the patient. ${ }^{1,5,13}$ Patients at risk can be followed more closely, and spasticity may be treated both pharmacologically and non-pharmacologically. ${ }^{30}$ It is uncertain whether early treatment reduces spasticity in the long term. However, it has been well-established that such treatments have led to significant improvements in spasticity-related impairments, motor function, and quality of life among patients poststroke. ${ }^{10,30-33}$

The second clinical implication may come from the finding that the assessment of spasticity at day 3 or 10 could not predict spasticity after 1 year. Although the univariate logistic regression showed a significant association between spasticity at day 10

\section{Comment: \\ How and why to predict spasticity after stroke?}

Although many stroke patients present with spasticity, this impairment remains a riddle for physicians. Why, when, and how does a patient develop spasticity, whereas another patient with a similar cerebral lesion does not? Moreover, the evolution of spasticity among these chronic patients and its relation to functional activity are not straightforward. Thus, the assessment and treatment of spasticity remain a challenge in neurorehabilitation.

Opheim et al. ${ }^{1}$ identify the early predictors of spasticity among stroke patients: age, sex, and neurologic impairments assessed with the Fugl-Meyer scale. Assessing the patient 10 days and 4 weeks after stroke allows the prediction, respectively, of the presence of spasticity and its severity at 1 year poststroke. Interestingly, stroke severity assessed by the NIH Stroke Scale at admission was not a predictor. This emphasizes the importance of assessing patients regularly and accurately during rehabilitation. Ideally, this assessment should not focus only on neurologic impairments. Following the WHO International Classification of Functioning, Disability, and Health (www.who.int/ classifications/icf), the activities that the patient performs in his or her environment and his or her social participation should also be assessed.

This study also underlines the usefulness of the Fugl-Meyer scale. However, whereas the authors used the original ordinal scale, they submitted the results to complex statistical methods. The Fugl-Meyer scale, as many other scales used in neurorehabilitation, ${ }^{2}$ has been transformed to a linear scale through Rasch analysis. In clinical practice and future research, it would be preferable to use these improved versions to optimize the quality of assessment and to gather continuous data suitable to powerful parametric statistics. ${ }^{3,4}$

Early identification of patients at risk of developing spasticity should improve the quality of care. They should be regularly assessed and would benefit from early treatment to avoid long-term complications (e.g., contractures), especially for the most impaired patients or those with reduced access to specialists.

1. Opheim A, Danielsson A, Alt Murphy M, Persson HC, Sunnerhagen KS. Early prediction of long-term upper limb spasticity after stroke: part of the SALGOT study. Neurology 2015;85:873-880.

2. Belvedere SL, Morton NA. Application of Rasch analysis in health care is increasing and is applied for variable reasons in mobility instruments. J Clin Epidemiol 2010;63:1-11.

3. Grimby G, Tennant A, Tesio L. The use of raw scores from ordinal scales: time to end malpractice? J Rehabil Med 2012;44:97-98.

4. Smith AG, Burns TM. Clinical measurement tools in therapeutic trials: time to make a Rasch decision? Neurology 2014;83:2104-2105.

Thierry M. Lejeune, $M D, P h D$

Gaëtan Stoquart, $M D, P h D$

From the Physical Medicine and Rehabilitation Department, Cliniques Universitaires Saint-Luc, Université Catholique de Louvain, Brussels, Belgium.

Study funding: No targeted funding reported.

Disclosure: The authors report no disclosures relevant to the manuscript. Go to Neurology.org for full disclosures. and spasticity 12 months poststroke, this association was lost in the multivariate regression analysis, as the FMA-UE was a much stronger predictor. Only after 4 weeks was the presence of spasticity a predictor, as patients with spasticity had a 30 times higher OR for severe spasticity 12 months poststroke than those without spasticity. Additionally, the chance of developing severe spasticity 1 year after stroke was very low if the assessment of spasticity at week 4 showed no spasticity. Consequently, there was a time point between 10 and 28 days poststroke at which spasticity became a predictor for severe spasticity, indicating that spasticity predominantly emerged and became manifest during this period. Therefore, the clinical value of assessing spasticity in patients much earlier than 4 weeks poststroke to predict long-term severe spasticity may be limited. However, the assessment of spasticity at this time may be important for other purposes. Both of these clinical implications indicate a need for regular and structured follow-ups for patients poststroke $\mathrm{e}^{34}$ as spasticity and related impairments may develop over months.

The assessment of spasticity may be a limitation as the MAS is an ordinal, clinical assessment scale and not a metric measure of spasticity. However, it does not require any equipment, is easy to apply in different settings, is frequently used, and has relatively good intrarater reliability. ${ }^{21}$ In the present study, MAS was dichotomized for both any spasticity and severe spasticity, which may be claimed to be arbitrary and not necessarily coincide with important clinical divisions. The dichotomization for any spasticity has shown that patients with spasticity had poorer sensorimotor function, more pain, and reduced ROM than those without spasticity. ${ }^{5}$

In the current study, the summed score of the ordinal FMA-UE scale was used in the prediction models, which can be a limitation. However, the FMA-UE has been shown to have excellent psychometric properties, to be a valid indicator of motor recovery, and is widely used to indicate stroke severity. ${ }^{35-37}$ The unidimensional hierarchy of the FMA-UE has been demonstrated both in acute and chronic stroke $e^{37,38}$ and as nonlinearity of the FMA-UE could not be shown, we chose to use the FMA-UE in the analysis.

The patients in the present study may be regarded as fairly representative for patients with first stroke, with reduced arm function at day 3, living in a western European country, and receiving modern stroke care according to evidence-based practice. The patients in the present study may not be representative of the global population of persons poststroke.

\section{AUTHOR CONTRIBUTIONS}

Dr. Opheim conducted the analyses and wrote the drafts and the revisions of the manuscript. Dr. Danielsson developed the study design 
and commented on and critically reviewed manuscript drafts and revisions. Dr. Alt Murphy developed the study design, collected the data, and commented on and critically reviewed manuscript drafts and revisions. H.C. Persson developed the study design, collected the data, and commented on and critically reviewed manuscript drafts and revisions. Dr. Sunnerhagen developed the study design and commented on and critically reviewed manuscript drafts and revisions.

\section{ACKNOWLEDGMENT}

The authors thank the patients for participation, Eva-Lena Bustrén for helping with data collection and entry, and The Riks-Stroke Collaboration for data on smoking.

\section{STUDY FUNDING}

Funded in part by the Swedish Research Council (VR K 2012-70X-2212201-3), the Foundation of the Swedish National Stroke Association, the Health \& Medical Care Committee of the Regional Executive Board, Region Västra Götaland, an unconditional grant from Allergan, the Local Research and Development Board for Gothenburg, Södra Bohuslän, the Norrbacka-Eugenia Foundation, and the Promobilia Foundation.

\section{DISCLOSURE}

A. Opheim reports no disclosures relevant to the manuscript. A. Danielsson received payment from Allergan Norden $\mathrm{AB}$ for a lecture on physiotherapy in spasticity and from Camp Scandinavia for travel costs to a rehabilitation meeting as payment for a lecture on physiotherapy. M. Murphy and H. Persson report no disclosures relevant to the manuscript. K. Sunnerhagen works for the National Board of Health and Welfare regarding guidelines for Stroke. She has talked about these guidelines at a meeting sponsored by Pfizer. She has been invited to a meeting by Allergan Europe to discuss outcome measures; poststroke checklist. Go to Neurology.org for full disclosures.

Received November 6, 2014. Accepted in final form April 9, 2015.

\section{REFERENCES}

1. Brainin M, Norrving B, Sunnerhagen KS, et al. Poststroke chronic disease management: towards improved identification and interventions for poststroke spasticity-related complications. Int J Stroke 2011;6:42-46.

2. Zorowitz RD, Gillard PJ, Brainin M. Poststroke spasticity: sequelae and burden on stroke survivors and caregivers. Neurology 2013;80:S45-S52.

3. Lundström E, Smits A, Borg J, Terént A. Four-fold increase in direct costs of stroke survivors with spasticity compared with stroke survivors without spasticity: the first year after the event. Stroke 2010;41:319-324.

4. Brainin M. Poststroke spasticity: treating to the disability. Neurology 2013;80:S1-S4.

5. Opheim A, Danielsson A, Alt Murphy M, Persson HC, Sunnerhagen KS. Upper limb spasticity during the first year after stroke: a longitudinal study at the University of Gothenburg (SALGOT). Am J Phys Med Rehabil 2014;93:884-896.

6. Lundström E, Terént A, Borg J. Prevalence of disabling spasticity 1 year after first-ever stroke. Eur J Neurol 2008; 15:533-539.

7. Watkins CL, Leathley MJ, Gregson JM, Moore AP, Smith TL, Sharma AK. Prevalence of spasticity post stroke. Clin Rehabil 2002;16:515-522.

8. Urban PP, Wolf T, Uebele M, et al. Occurrence and clinical predictors of spasticity after ischemic stroke. Stroke 2010;41:2016-2020.

9. Lundstrom E, Smits A, Terent A, Borg J. Time-course and determinants of spasticity during the first six months following first-ever stroke. J Rehabil Med 2010;42:296-301.
10. Wissel J, Manack A, Brainin M. Toward an epidemiology of poststroke spasticity. Neurology 2013;80:S13-S19.

11. Kong KH, Lee J, Chua KS. Occurrence and temporal evolution of upper limb spasticity in stroke patients admitted to a rehabilitation unit. Arch Phys Med Rehabil 2012;93:143-148.

12. Leathley MJ, Gregson JM, Moore AP, Smith TL, Sharma AK, Watkins CL. Predicting spasticity after stroke in those surviving to 12 months. Clin Rehabil 2004;18:438-443.

13. Sunnerhagen KS, Olver J, Francisco GE. Assessing and treating functional impairment in poststroke spasticity. Neurology 2013;80:S35-S44.

14. Alt Murphy M, Persson HC, Danielsson A, Broeren J, Lundgren-Nilsson A, Sunnerhagen KS. SALGOT: Stroke Arm Longitudinal study at the University of Gothenburg, prospective cohort study protocol. BMC Neurol 2011;11:56.

15. Tunstall-Pedoe $\mathrm{H}$; for the WHO MONICA Project. The World Health Organization MONICA Project (monitoring trends and determinants in cardiovascular disease): a major international collaboration. J Clin Epidemiol 1988;41:105-114.

16. Nordin A, Alt Murphy M, Danielsson A. Intra-rater and inter-rater reliability at the item level of the Action Research Arm Test for patients with stroke. J Rehabil Med 2014;46:738-745.

17. Bamford J, Sandercock P, Dennis M, Burn J, Warlow C. Classification and natural history of clinically identifiable subtypes of cerebral infarction. Lancet 1991;337:1521-1526.

18. Adams HP Jr, Bendixen BH, Kappelle LJ, et al. Classification of subtype of acute ischemic stroke: definitions for use in a multicenter clinical trial: TOAST: Trial of Org 10172 in Acute Stroke Treatment. Stroke 1993;24:35-41.

19. Brott T, Adams HP, Olinger CP, et al. Measurements of acute cerebral infarction: a clinical examination scale. Stroke 1989;20:864-870.

20. Fugl-Meyer AR, Jaasko L, Leyman I, Olsson S, Steglind S. The post-stroke hemiplegic patient: 1: a method for evaluation of physical performance. Scand J Rehabil Med 1975;7:13-31.

21. Bohannon RW, Smith MB. Interrater reliability of a modified Ashworth Scale of muscle spasticity. Phys Ther 1987; 67:206-207.

22. Welmer AK, Widen Holmqvist L, Sommerfeld DK. Location and severity of spasticity in the first $1-2$ weeks and at 3 and 18 months after stroke. Eur J Neurol 2010;17:720-725.

23. Cox DR, Wermuth N. Tests of linearity, multivariate normality and the adequacy of Linear scores. J R Stat Soc Ser C Appl Stat 1994;43:347-355.

24. Pallant J. SPSS Survival Manual, 2nd ed. Berkshire, UK: Open University Press; 2005.

25. Vandenbroucke JP, von Elm E, Altman DG, et al. Strengthening the Reporting of Observational Studies in Epidemiology (STROBE): explanation and elaboration. Epidemiology 2007;18:805-835.

26. von Elm E, Altman DG, Egger M, et al. The Strengthening the Reporting of Observational Studies in Epidemiology (STROBE) statement: guidelines for reporting observational studies. Epidemiology 2007;18:800-804.

27. Chung SG, Van Rey EM, Bai Z, Rogers MW, Roth EJ, Zhang LQ. Aging-related neuromuscular changes characterized by tendon reflex system properties. Arch Phys Med Rehabil 2005;86:318-327.

28. Peduzzi P, Concato J, Kemper E, Holford TR, Feinstein AR. A simulation study of the number of events per variable in logistic regression analysis. J Clin Epidemiol 1996;49:1373-1379. 
29. Thilmann AF, Fellows SJ, Garms E. The mechanism of spastic muscle hypertonus: variation in reflex gain over the time course of spasticity. Brain 1991;114:233-244.

30. Thibaut A, Chatelle C, Ziegler E, Bruno MA, Laureys S, Gosseries O. Spasticity after stroke: physiology, assessment and treatment. Brain Inj 2013;27:1093-1105.

31. Ward AB. A summary of spasticity management: a treatment algorithm. Eur J Neurol 2002;9:48-52.

32. Cousins E, Ward A, Roffe C, Rimington L, Pandyan A. Does low-dose botulinum toxin help the recovery of arm function when given early after stroke? A phase II randomized controlled pilot study to estimate effect size. Clin Rehabil 2010;24:501-513.

33. Ward AB. A literature review of the pathophysiology and onset of post-stroke spasticity. Eur J Neurol 2012;19:21-27.

34. Philp I, Brainin M, Walker MF, et al. Development of a poststroke checklist to standardize follow-up care for stroke survivors. J Stroke Cerebrovasc Dis 2013;22:e173-e180.
35. Gor-García-Fogeda MD, Molina-Rueda F, CuestaGómez A, Carratalá-Tejada M, Alguacil-Diego IM, Miangolarra-Page JC. Scales to assess gross motor function in stroke patients: a systematic review. Arch Phys Med Rehabil 2014;95:1174-1183.

36. Salter K, Campbell N, Richardson M, et al. Outcome Measures in Stroke Rehabilitation: Evidence Reviews [Serial Online]. 2013:1-144. Available at: http://www.ebrst.com/ sites/default/files/Chapter21_Outcome-Measures_FINAL_ 16ed.pdf. Accessed April 3, 2015.

37. Crow JL, Kwakkel G, Bussmann JB, Goos JA, Harmelingvan der Wel BC. Are the hierarchical properties of the Fugl-Meyer assessment scale the same in acute stroke and chronic stroke? Phys Ther 2014;94:977-986.

38. Crow JL, Harmeling-van der Wel BC. Hierarchical properties of the motor function sections of the Fugl-Meyer assessment scale for people after stroke: a retrospective study. Phys Ther 2008;88:1554-1567.

\section{This Week's Neurology ${ }^{\circledR}$ Podcast}

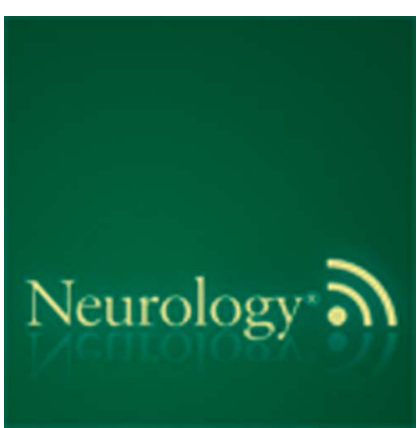

Anti-DPPX encephalitis: Pathogenic effects of antibodies on gut and brain neurons (see p. 890)

This podcast begins and closes with Dr. Robert Gross, Editor-inChief, briefly discussing highlighted articles from the September 8, 2015, issue of Neurology. In the second segment, Dr. Michelle Fullard talks with Johannes Piepgras about his paper on pathogenic effects of antibodies on gut and brain neurons in anti-DPPX encephalitis. In our newest feature, "What's Trending," Dr. Ted Burns interviews Morgan Sorenson about Twitter handles. In the next part of the podcast, Dr. Andy Southerland focuses his interview with Dr. Kevin Barrett on the topic of teleneurology (nonstroke applications).

Disclosures can be found at Neurology.org.

At Neurology.org, click on "RSS" in the Neurology Podcast box to listen to the most recent podcast and subscribe to the RSS feed.

CME Opportunity: Listen to this week's Neurology Podcast and earn 0.5 AMA PRA Category 1 CME Credits ${ }^{\mathrm{TM}}$ by answering the multiple-choice questions in the online Podcast quiz.

\section{Get Connected. Stay Connected.}

Connect with the American Academy of Neurology's popular social media channels to stay up-todate on the latest news and breakthroughs in neurology, and network with peers and neurology thought leaders. Visit AAN.com/Connect. 


\section{Neurology}

Early prediction of long-term upper limb spasticity after stroke: Part of the SALGOT study

Arve Opheim, Anna Danielsson, Margit Alt Murphy, et al. Neurology 2015;85;873-880 Published Online before print August 14, 2015

DOI 10.1212/WNL.0000000000001908

This information is current as of August 14, 2015

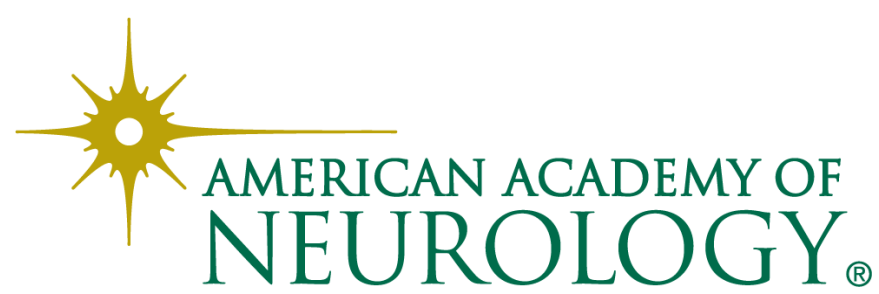




\section{Updated Information \& Services}

\section{Supplementary Material}

\section{References}

Citations

Subspecialty Collections

\section{Permissions \& Licensing}

\section{Reprints}

including high resolution figures, can be found at:

http://n.neurology.org/content/85/10/873.full

Supplementary material can be found at: http://n.neurology.org/content/suppl/2015/08/14/WNL.0000000000001 908.DC2

http://n.neurology.org/content/suppl/2015/08/14/WNL.0000000000001 908.DC1

This article cites 36 articles, 11 of which you can access for free at: http://n.neurology.org/content/85/10/873.full\#ref-list-1

This article has been cited by 1 HighWire-hosted articles: http://n.neurology.org/content/85/10/873.full\#\#otherarticles

This article, along with others on similar topics, appears in the following collection(s):

\section{All Cerebrovascular disease/Stroke}

http://n.neurology.org/cgi/collection/all_cerebrovascular_disease_strok e

\section{All Rehabilitation}

http://n.neurology.org/cgi/collection/all_rehabilitation

\section{Cohort studies}

http://n.neurology.org/cgi/collection/cohort_studies

\section{Prognosis}

http://n.neurology.org/cgi/collection/prognosis

Information about reproducing this article in parts (figures,tables) or in its entirety can be found online at:

http://www.neurology.org/about/about_the_journal\#permissions

Information about ordering reprints can be found online: http://n.neurology.org/subscribers/advertise

Neurology ${ }^{\circledR}$ is the official journal of the American Academy of Neurology. Published continuously since 1951, it is now a weekly with 48 issues per year. Copyright (C) 2015 American Academy of Neurology. All rights reserved. Print ISSN: 0028-3878. Online ISSN: 1526-632X.

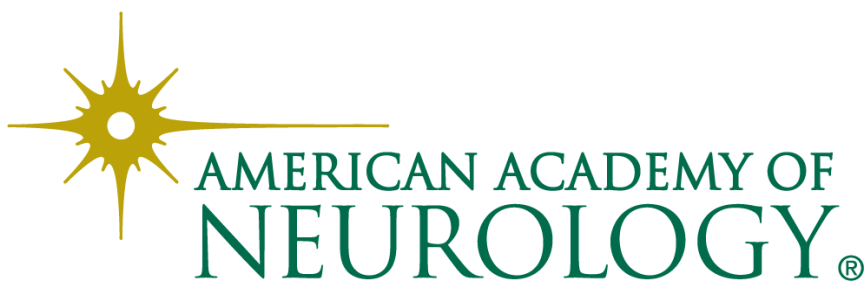

